\title{
ON THE DISTRIBUTION (MOD 1) OF POLYNOMIALS OF A PRIME VARIABLE
}

\author{
MING-CHIT LIU AND KAI-MAN TSANG
}

\section{§1. Introduction}

Throughout, $\varepsilon$ is any small positive number, $\theta$ any real number, $n$, $n_{j}, k, N$ some positive integers and $p, p_{j}$ any primes. By $\|\theta\|$ we mean the distance from $\theta$ to the nearest integer. Write $C(\varepsilon), C(\varepsilon, k)$ for positive constants which may depend on the quantities indicated inside the parentheses.

Dirichlet's theorem says that for any $\theta, N$ there exists $n$ such that

$$
n \leqslant N \text { and }\|\theta n\|<N^{-1} .
$$

Furthermore, as a direct consequence of (1.1), there are infinitely many $n$ such that

$$
\|\theta n\|<n^{-1} \text {. }
$$

Improving an estimate of Vinogradov [12], Heilbronn [6] extended (1.1) by showing that for any $\theta, \varepsilon, N$ there are $n$ and $C(\varepsilon)$ such that

$$
n \leqslant N \text { and }\left\|\theta n^{2}\right\|<C(\varepsilon) N^{-1 / 2+\varepsilon} .
$$

Later, Davenport [3] extended (1.3) by proving that if $g$ is a polynomial of degree $k \geqslant 2$ with real coefficients and without constant term then for any $\varepsilon, N$ there are $n$ and $C(\varepsilon, k)$ such that

$$
n \leqslant N \text { and }\|g(n)\|<C(\varepsilon, k) N^{-1 / 2^{k-1)+e}} .
$$

The results of Heilbronn [6] and Davenport [3] sparked off a series of investigations (see [9]). In particular, recently Schmidt has made remarkable progress in $[9,10]$. However all these developments concerning (1.1) have no parallel results for prime. This can be seen from the following example. Let $q$ be any positive integer having at least two

Received June 6, 1980. 
distinct prime factors and $\left\{\alpha_{j}\right\}_{1}^{\infty}$ a sequence of irrationals which converges to the rational $a / q$ with $(a, q)=1$. Obviously

$$
\left\|p^{k} a / q\right\| \geqslant 1 / q
$$

for any prime $p$. Suppose that when $\theta$ is irrational, (1.4) has a parallel result for prime, i.e. for any $\alpha_{j}, \varepsilon, N$ there are $p$ and $C(\varepsilon, k)$ such that

$$
p \leqslant N \text { and }\left\|\alpha_{j} p^{k}\right\|<C(\varepsilon, k) N^{-\delta+\varepsilon},
$$

where $\varepsilon<\delta$ and $\delta$ depends on $k$ only. Now if $N^{\delta-\varepsilon}>q(C(\varepsilon, k)+1)$ and $\alpha_{j}$ satisfies $\left|\alpha_{j}-a / q\right|<N^{-k-\delta}$, then by (1.6),

$$
\left\|p^{k} a / q\right\| \leqslant\left\|\alpha_{j} p^{k}\right\|+p^{k}\left\|\alpha_{j}-a / q\right\|<N^{-\delta+\varepsilon}(C(\varepsilon, k)+1)<1 / q .
$$

This contradicts (1.5).

On the contrary, concerning (1.2) there is indeed a parallel result for prime. It was mentioned in [5] that by a result of Vinogradov [14, Chapter 9] for any $\varepsilon$ and irrational $\alpha$, there are infinitely many primes $p$ such that $\|\alpha p\|<p^{-1 / 5+\varepsilon}$. Recently this inequality was improved by Vaughan [11] to $\|\alpha p\|<p^{-1 / 4}(\log p)^{8}$. The object of our present paper is to extend Dirichlet's theorem (1.2) to polynomials of a prime variable as that (1.3) and (1.4) extend Dirichlet's theorem (1.1). We shall prove

THEOREM. If $f$ is any polynomial of degree $k \geqslant 2$ with real coefficients and irrational leading coefficient then for any $\varepsilon>0$ there are infinitely many primes $p$ such that

$$
\|f(p)\|<p^{-A(k)+\varepsilon}
$$

where $A(k)=\left(3(k+1) 4^{k+1}\right)^{-1}$.

By (1.5) we see that the irrationality in our theorem is essential. In our proof, unlike most previous work in this field, we make no use of the Heilbronn argument [6] presented by Davenport in [3] but we modify an earlier method due to Davenport and Heilbronn [1]. Also in $\S 4$ we are able to use the full-strength of a result of Vinogradov [13] which determines the exponent, $A(k)$ in our Theorem.

\section{§2. Notation}

Let $\delta$ be a small positive number $(<1)$ and $x$ a real variable. Write $e(x)=\exp (i 2 \pi x)$ and denote the integral part of $x$ by $[x]$. Let $\alpha$ be the 
leading coefficient of the given polynomial $f$. Since $\alpha$ is irrational, by Theorem 183 [4] there are infinitely many convergents $a / q$ such that

$$
|\alpha-a / q|<\left(2 q^{2}\right)^{-1} .
$$

For sufficiently large $q$, put

$$
\begin{gathered}
X=q^{1 /(k-2 / 3)}, \quad L=\log X \\
I_{j}(x)=\int_{X}^{2 X} e\left((-1)^{j} x y^{k}\right) d y \quad(j=1,2), \\
\left\{\begin{array}{l}
S_{j}(x)=\sum_{x<n \leqslant 2 X} e\left((-1)^{j} x n^{k}\right) \quad(j=1,2), \\
S_{3}(x)=\sum_{i x<p \leqslant 2 \delta X} e(x f(p)), \quad S_{4}(x)=\sum_{\delta X<p \leqslant 2 \delta X} e\left(x p^{k}\right), \\
S_{j}(x)=\sum_{\delta . X<n \leqslant 2 \delta X} e\left(x n^{k}\right) \quad(j=5,6, \cdots, s),
\end{array}\right.
\end{gathered}
$$

where

$$
s=2^{k}+2
$$

Trivially,

$$
\left|S_{\jmath}(x)\right| \leqslant X(j=1, \cdots, s) \text { and }\left|I_{j}(x)\right| \leqslant X(j=1,2) .
$$

Furthermore we put

$$
\begin{gathered}
V(x)=\prod_{j=1}^{s} S_{j}(x), \quad W(x)=I_{1}(x) I_{2}(x) \prod_{j=3}^{s} S_{j}(x), \\
A(k)=\left(3(k+1) 4^{k+1}\right)^{-1}, \\
\tau=X^{-A(k)+\varepsilon},
\end{gathered}
$$

$$
K_{\tau}(x)= \begin{cases}\tau^{2} & \text { if } x=0 \\ \left(\frac{\sin \pi \tau x}{\pi x}\right)^{2} & \text { otherwise }\end{cases}
$$

Obviously,

$$
K_{\tau}(x) \leqslant \tau^{2}
$$

We partition the real line into

$$
\left\{\begin{array}{l}
E_{1}=\left\{x:|x| \leqslant X^{-k+1 / 3}\right\} \\
E_{2}=\left\{x: X^{-k+1 / 3}<|x| \leqslant X^{2 A(k)}\right\}, \\
E_{3}=\left\{x: X^{2 A(k)}<|x|\right\}
\end{array}\right.
$$


If $Y>0$ we use $Z \ll Y$ (or $Y \gg Z$ ) to denote $|Z|<C Y$ where $C$ is some positive constant. The constants implied by $O, \ll, \gg$ may depend on the given constants, $k, \varepsilon, \delta$ and the coefficients of $f$ only.

\section{$\S 3$. Integration over $E_{1}$}

LEMMa 1. For any real y we have

$$
\int_{-\infty}^{\infty} e(x y) K_{\tau}(x) d x=\max (0, \tau-|y|) .
$$

Proof. See Lemma 2 in [8].

LemMa 2. We have

$$
\int_{-\infty}^{\infty} W(x) K_{\tau}(x) d x \gg \tau^{2} X^{s-k} L^{-2}
$$

Proof. Let $B$ denote the cartesian product of the intervals, $X^{k} \ll y_{j}$ $\ll(2 X)^{k}(j=1,2)$ and let the set $B^{*}$ of $\left(y_{1}, y_{2}\right)$ be defined by the following (3.1), (3.2), (3.3) and (3.4).

$$
\begin{gathered}
2 X^{k} \leqslant y_{1} \leqslant 3 X^{k}, \\
y_{2}=y_{1}+\phi-f\left(p_{3}\right)-p_{4}^{k}-\sum_{5 \leqslant j \leqslant s} n_{j}^{k},
\end{gathered}
$$

where

$$
\delta X \leqslant p_{3}, p_{4}, n_{5}, \cdots, n_{s} \leqslant 2 \delta X
$$

and $\phi$ is a real variable satisfying

$$
|\phi|<\tau / 2 .
$$

By (3.1), (3.2), (3.3) and (3.4) we see that

$$
y_{2} \leqslant 3 X^{k}+\tau / 2+2|\alpha|(2 \delta X)^{k}+(2 \delta X)^{k}+(s-4)(2 \delta X)^{k}<4 X^{k} .
$$

Similarly $y_{2}>X^{k}$. So

$$
B^{*} \subset B
$$

By (2.6), (2.2) and (2.3) we have

$$
\begin{aligned}
\int_{-\infty}^{\infty} W(x) K_{\tau}(x) d x=\sum_{1} \int_{-\infty}^{\infty} & \left(\prod_{j=1}^{2} \int_{X^{k}}^{(2 X)^{k}}\left(k y_{j}^{1-1 / k}\right)^{-1} e\left((-1)^{j} x y_{j}\right) d y_{j}\right) \\
& \times e\left(x\left\{f\left(p_{3}\right)+p_{4}^{k}+\sum_{5 \leqslant j \leqslant s} n_{j}^{k}\right\}\right) K_{\tau}(x) d x
\end{aligned}
$$


where $\sum_{1}$ is a summation taken over all $p_{j}, n_{j}$ satisfying (3.3). Then by Lemma 1, (3.5), (3.2), (3.4) and (3.1) we have

$$
\begin{aligned}
\int_{-\infty}^{\infty} W(x) K_{\tau}(x) d x & \gg X^{2(1-k)} \sum_{1} \int_{B} \max \left(0, \tau-\mid-y_{1}+y_{2}+f\left(p_{3}\right)+p_{4}^{k}\right. \\
& \left.+\sum_{5 \leqslant j \leqslant s} n_{j}^{k} \mid\right) d y_{1} d y_{2} \\
& \gg X^{2(1-k)} \sum_{1}(\tau-(\tau / 2)) \int_{B^{*}} d y_{1} d y_{2}(\phi) \\
& \gg X^{2(1-k)} \sum_{1} \tau\left(\tau X^{k}\right) \\
& \gg \tau^{2} X^{2-k} X^{s-4}(X / L)^{2} .
\end{aligned}
$$

The last inequality follows from (3.3) and the prime number theorem. This proves Lemma 2.

Lemma 3. If $|x| \ll X^{-k+1 / 3}$ then for $j=1,2$

$$
S_{j}(x)=I_{j}(x)+O(1) .
$$

Proof. This is essentially the Corollary in [2, p. 85].

LEMma 4. We have

$$
\int_{E_{1}} V(x) K_{\tau}(x) d x \gg \tau^{2} X^{s-k} L^{-2}
$$

Proof. By (2.6), Lemma 3 and (2.5) we have, when $x \in E_{1}$

$$
\begin{aligned}
|V(x)-W(x)| & =\left|S_{1}\left(S_{2}-I_{2}\right)+I_{2}\left(S_{1}-I_{1}\right)\right| \prod_{j=3}^{s}\left|S_{j}(x)\right| \\
& =O(X) \prod_{j=3}^{s}\left|S_{j}(x)\right|=O\left(X^{s-1}\right) .
\end{aligned}
$$

So in view of (2.9) and (2.10)

$$
\left|\int_{E_{1}} V(x) K_{\tau}(x) d x-\int_{E_{1}} W(x) K_{\tau}(x) d x\right| \ll \tau^{2} X^{s-1} \int_{E_{1}} d x \ll \tau^{2} X^{s-k-2 / 3} .
$$

On the other hand, by integration by parts and (2.2) if $x \neq 0$ we have

$$
I_{j}(x)=O\left(|x|^{-1} X^{-k+1}\right) \text {. }
$$

It follows from (2.9), (3.7), (2.5) and (2.10) that

$$
\int_{x \notin E_{1}} W(x) K_{\tau}(x) d x \ll \tau^{2} X^{s-2} X^{2(1-k)} \int_{x \notin E_{1}}|x|^{-2} d x \ll \tau^{2} X^{s-k-1 / 3} .
$$

Lemma 4 follows from Lemma 2, (3.6) and (3.8). 


\section{§4. Integration over $E_{2}$}

Lemma 5. Let $\lambda_{3}=\alpha$ (the leading coefficient of $f$ ) and $\lambda_{4}=1$. Suppose that for $j=3$ or 4 there are integers $a_{j}, q_{j}$ with $\left(a_{j}, q_{j}\right)=1,1 \leqslant q_{j}$ and

$$
\left|\lambda_{j} x-a_{j}\right| q_{j} \mid \leqslant q_{j}^{-2} .
$$

If

$$
Q=\min \left(q_{j},[2 \delta X]^{k} / q_{j}\right), \quad U=\min \left(Q,[2 \delta X]^{1 / 3}\right)
$$

and

$$
Q \geqslant(k \log [2 \delta X])^{(2 k+1) 4^{3 k-1}}
$$

then

$$
S_{j}(x) \ll X U^{-3 A(k)},
$$

where $A(k)$ is defined in (2.7).

Proof. This is the Theorem in [13].

Lemma 6. We have

$$
\sup _{x \in E_{2}} \min \left(\left|S_{3}(x)\right|,\left|S_{4}(x)\right|\right) \ll X^{1-A(k)} .
$$

Proof. Let $\lambda_{3}=\alpha$, which is the leading coefficient of the polynomial $f$, and $\lambda_{4}=1$. By Dirichlet's theorem [4, p. 30] for each $x \in E_{2}$ there are integers $a_{j}, q_{j}$ with $\left(a_{j}, q_{j}\right)=1$ and

$$
1 \leqslant q_{j} \leqslant \delta^{-1} X^{k-1 / 3}
$$

such that

$$
\left|\lambda_{j} x-a_{j}\right| q_{j} \mid \leqslant \delta X^{-k+1 / 3} q_{j}^{-1} \quad(j=3,4) .
$$

By the same argument as that in Lemma 13 of [8] we can prove that $\max \left(q_{3}, q_{4}\right) \geqslant X^{1 / 3}$. In the proof we need (2.1), that is the irrationality of $\alpha$. Then Lemma 6 follows from Lemma 5 .

Lemma 7. For $j \neq 3,4$ we have

$$
\int_{-\infty}^{\infty}\left|S_{j}(x)\right|^{2 k} K_{z}(x) d x \ll \tau X^{2^{k}-k} L^{c},
$$

where $c$ is some positive constant depending on $k$ only.

Proof. This is a consequence of Hua's Lemma [Theorem 4, 7]. See Lemma 21 in [8]. 
LEMMA 8. We have

$$
\int_{E_{2}}|V(x)| K_{\tau}(x) d x \ll \tau^{2} X^{s-k} L^{-3} .
$$

Proof. By Lemma 6 we have

$$
\begin{aligned}
\int_{E_{2}}|V(x)| K_{\tau}(x) d x \leqslant & \sup _{x \in E_{2}} \min \left(\left|S_{3}(x)\right|,\left|S_{4}(x)\right|\right) \\
& \times\left\{\int_{E_{2}}\left(\left|S_{3}(x)\right|+\left|S_{4}(x)\right|\right)\left|\prod_{j \neq 3,4} S_{j}(x)\right| K_{\tau}(x) d x\right\} \\
& \ll X^{1-A(k)}\left\{J_{1}+J_{2}\right\}, \quad \text { say. }
\end{aligned}
$$

Note that by (2.4) there are $2^{k}$ factors in the above product $\prod_{j \neq 3,4} S_{j}(x)$. We denote the products taken over the first $2^{k-1}$ and last $2^{k-1}$ factors by $\prod_{1}$ and $\prod_{2}$ respectively. By (2.5) and Hölder's inequality we have

$$
\begin{aligned}
J_{1} & \ll X \int_{E_{2}}\left|\prod_{j \neq 3,4} S_{j}(x)\right| K_{\tau}(x) d x \\
& \ll X\left\{\prod_{1}\left(\int_{-\infty}^{\infty}\left|S_{j}(x)\right|^{2 k} K_{\tau}(x) d x\right)^{21-k}\right\}^{1 / 2}\left\{\prod_{2}\left(\int_{-\infty}^{\infty}\left|S_{j}(x)\right|^{2^{k}} K_{\tau}(x) d x\right)^{21-k}\right\}^{1 / 2} .
\end{aligned}
$$

The same argument holds for $J_{2}$, then by Lemma 7 we have

$$
J_{1} \text { and } J_{2} \ll \tau X^{2^{k-k+1}} L^{c} .
$$

This, together with (4.4), (2.4) and (2.8), proves Lemma 8.

\section{$\S 5$. Completion of the proof}

Lemma 9. Let $\Omega(x)=\sum e\left(x \omega\left(y_{1}, \cdots, y_{n}\right)\right)$, where $\omega$ is any real-valued function and the summation is over any finite set of values $y_{1}, \cdots, y_{n}$. Then for any $R>4 / \tau$ we have

$$
\int_{|x|>R}|\Omega(x)|^{2} K_{\tau}(x) d x \ll(R \tau)^{-1} \int_{-\infty}^{\infty}|\Omega(x)|^{2} K_{\tau}(x) d x .
$$

Proof. This follows from Lemma 2 in [2]. See Lemma 16 in [8].

LEMma 10. We have

$$
\int_{E_{3}}|V(x)| K_{\tau}(x) d x \ll \tau^{2} X^{s-k} L^{-3}
$$

Proof. By (2.5), Lemma 9 with $R=X^{2 A(k)}$ and a similar argument as in the proof of Lemma 8 , we have 


$$
\begin{aligned}
\int_{E_{3}}|V(x)| K_{\tau}(x) d x \ll X^{2}\left(X^{2 A(k)} \tau\right)^{-1}\{ & \left.\prod_{1}\left(\int_{-\infty}^{\infty}\left|S_{j}(x)\right|^{2 k} K_{\tau}(x) d x\right)^{2^{1-k}}\right\}^{1 / 2} \\
& \times\left\{\prod_{2}\left(\int_{-\infty}^{\infty}\left|S_{\jmath}(x)\right|^{2^{k}} K_{\tau}(x) d x\right)^{2^{1-k}}\right\}^{1 / 2} \\
\ll \tau^{2} X^{s-k} L^{-3} . &
\end{aligned}
$$

This proves Lemma 10.

We come now to the proof of our Theorem. By Lemma 1 we have

$$
J=\int_{-\infty}^{\infty} V(x) K_{\tau}(x) d x=\sum_{2} \max \left(0, \tau-\left|n_{1}^{k}-n_{2}^{k}+f\left(p_{3}\right)+p_{4}^{k}+\sum_{5 \leqslant j \leqslant s} n_{j}^{k}\right|\right),
$$

where the summation $\sum_{2}$ is taken over all $s$-tuples $\left(n_{1}, n_{2}, p_{3}, p_{4}, n_{5}, \cdots, n_{s}\right)$ lying in

$$
X \leqslant n_{1}, n_{2} \leqslant 2 X ; \quad \delta X \leqslant p_{3}, p_{4}, n_{5}, \cdots, n_{s} \leqslant 2 \delta X
$$

Then

$$
J \leqslant \tau N
$$

where $N$ is the number of $\left(n_{1}, n_{2}, p_{3}, p_{4}, n_{5}, \cdots, n_{s}\right)$ satisfying (5.1) and

$$
\left|n_{1}^{k}-n_{2}^{k}+f\left(p_{3}\right)+p_{4}^{k}+\sum_{5 \leqslant j \leqslant s} n_{j}^{k}\right|<\tau=X^{-A(k)+\varepsilon} .
$$

Now, by Lemmas 4,8 and 10 we have

$$
J=\sum_{\nu=1}^{3} \int_{E_{\nu}} V(x) K_{\tau}(x) d x \gg \tau^{2} X^{s-k} L^{-2} .
$$

So by (5.2)

$$
N \gg \tau X^{s-k} L^{-2} \longrightarrow \infty \quad \text { as } X \longrightarrow \infty \text {. }
$$

Since $n_{1}^{k}-n_{2}^{k}+p_{4}^{k}+\sum_{5 \leqslant j \leqslant s} n_{\jmath}^{k}$ is an integer and $\delta X \leqslant p_{3} \leqslant 2 \delta X$, by (5.3), (5.4) we see that

$$
\left\|f\left(p_{3}\right)\right\|<p_{3}^{-A(k)+\sigma}
$$

has infinitely many solutions in primes $p_{3}$. This proves our Theorem.

\section{References}

[1] H. Davenport and H. Heilbronn, On indefinite quadratic forms in five variables, J. London Math. Soc., 21 (1946), 185-193.

[2] H. Davenport and K. F. Roth, The solubility of certain diophantine inequalities, Mathematika, 2 (1955), 81-96. 
[ 3 ] H. Davenport, On a theorem of Heilbronn, Quart. J. Math. Oxford, (2), 18 (1967), $339-344$.

[4] G. H. Hardy and E. M. Wright, An Introduction to the Theory of Numbers, 4th ed. Oxford, 1965.

[5] S. Hartman and S. Knapowski, Bemerkungen über die Bruchteile von pa, Ann. Polon. Math., 3 (1957), 285-287.

[6] H. Heilbronn, On the distribution of the sequence $n^{2} \theta(\bmod 1)$, Quart. J. Math. Oxford, 19 (1948), 249-256.

[7] L. K. Hua, Additive Theory of Prime Numbers, Translations of Mathematical Monographs Vol. 13, Amer. Math. Soc., Providence, R.I. 1965.

[8] M. C. Liu, Approximation by a sum of polynomials involving primes, J. Math. Soc. Japan, 30 (1978), 395-412.

[9] W. M. Schmidt, Small Fractional Parts of Polynomials, Regional Conference Series in Mathematics No. 32, Amer. Math. Soc. Providence, R.I. 1977.

[10] - On the distribution modulo 1 of the sequence $\alpha n^{2}+\beta n$, Canad. J. Math., 29 (1977), 819-826.

[11] R. C. Vaughan, On the distribution of $\alpha p$ modulo 1, Mathematika, 28 $(1977), 135-$ 141.

[12] I. M. Vinogradov, Analytischer Beweis des Satzes über die Verteilung der Bruchteile eines ganzen Polynoms, (in Russian), Bull. Acad. Sci. USSR (6), 21 (1927), $567-578$.

[13] —, A new estimate of a trigonometric sum containing primes, (in Russian with English summary), Bull. Acad. Sci. USSR ser. Math., 2 (1938), 3-13.

[14] —- The Method of Trigonometrical Sums in the Theory of Numbers, New York: Interscience 1954.

Department of Mathematics

University of Hong Kong

Hong Kong 